\title{
Molecular Diagnosis of Hereditary Neuropathies by Whole Exome Sequencing and Expanding the Phenotype Spectrum
}

\author{
Sara Taghizadeh, MS ${ }^{1,2 *}$; Raheleh Vazehan, MS ${ }^{3 *}$; Maryam Beheshtian, MD, MPH ${ }^{1,3 *}$; Farnaz Sadeghinia, MS'; Zohreh Fattahi, PhD ${ }^{1,3}$; Marzieh \\ Mohseni, MS ${ }^{1,3}$; Sanaz Arzhangi, MS ${ }^{1}$; Shahriar Nafissi, MD'; Ariana Kariminejad, MD³ Hossein Najmabadi, PhD ${ }^{1,3^{*}}$; Kimia Kahrizi, MD ${ }^{1 *}$ \\ ${ }^{1}$ Genetics Research Center, University of Social Welfare and Rehabilitation Sciences, Tehran, Iran \\ ${ }^{2}$ Student Research Committee, University of Social Welfare and Rehabilitation Sciences, Tehran, Iran \\ ${ }^{3}$ Kariminejad - Najmabadi Pathology \& Genetics Center, Tehran, Iran \\ ${ }^{4}$ Department of Neurology, Shariati Hospital, Tehran University of Medical Sciences, Tehran, Iran
}

\begin{abstract}
Background: Inherited peripheral neuropathies (IPNs) are a group of neuropathies affecting peripheral motor and sensory neurons. Charcot-Marie-Tooth (CMT) disease is the most common disease in this group. With recent advances in next-generation sequencing (NGS) technologies, more than 100 genes have been implicated for different types of CMT and other clinically and genetically inherited neuropathies. There are also a number of genes where neuropathy is a major feature of the disease such as spinocerebellar ataxia (SCA) and hereditary spastic paraplegia (HSP). We aimed to determine the genetic causes underlying IPNs in Iranian families.

Methods: We performed whole exome sequencing (WES) for 58 PMP22 deletion-/duplication-negative unrelated Iranian patients with a spectrum of phenotypes and with a preliminary diagnosis of hereditary neuropathies.

Results: Twenty-seven (46.6\%) of the cases were genetically diagnosed with pathogenic or likely pathogenic variants. In this study, we identified genetically strong variants within genes not previously linked to any established disease phenotype in five (8.6\%) patients.

Conclusion: Our results highlight the advantage of using WES for genetic diagnosis in highly heterogeneous diseases such as IPNs. Moreover, functional analysis is required for novel and uncertain variants.

Keywords: Charcot-Marie-Tooth disease, Inherited peripheral neuropathy, Whole exome sequencing

Cite this article as: Taghizadeh S, Vazehan R, Beheshtian M, Sadeghinia F, Fattahi Z, Mohseni M, et al. Molecular diagnosis of hereditary neuropathies by whole exome sequencing and expanding the phenotype spectrum. Arch Iran Med. 2020;23(7):426433. doi: 10.34172/aim.2020.39.
\end{abstract}

Received: February 5, 2020, Accepted: April 8, 2020, ePublished: July 1, 2020

\section{Introduction}

Hereditary neuropathies comprise two groups of disorders: the first category is characterized by inherited peripheral neuropathies (IPNs). These are a group of neuropathies affecting peripheral motor and sensory neurons including hereditary motor and sensory neuropathy (HMSN) also known as Charcot-MarieTooth neuropathy (CMT), hereditary neuropathy with liability to pressure palsies, distal hereditary motor neuropathy, hereditary neuralgic amyotrophy, and hereditary sensory and autonomic neuropathy (HSAN) also known as hereditary sensory neuropathy (HSN). In the second category, peripheral neuropathy is part of hereditary neurological or multisystem disorders such as hereditary spastic paraplegias (HSPs), hereditary ataxia, mitochondrial disorders, disturbance of lipid metabolism such as trifunctional protein deficiency, porphyria, familial amyloid polyneuropathy or transthyretin amyloid polyneuropathy, giant axonal neuropathy, disorders with defective DNA, and miscellaneous conditions such as SeSAME (Seizures, Sensorineural deafness, Ataxia, Mental retardation, and Electrolyte imbalance) syndrome, Chediak-Higashi syndrome, etc. ${ }^{1}$

CMT is the most common IPN with a frequency of $1: 2,500$ and is caused by the degeneration of axon length or myelin sheath in peripheral neurons, leading to slowly progressive weakness and atrophy of distal muscles, reduced tendon reflexes, sensory loss, and limb deformities. ${ }^{2}$

At the present time, concurrent with technological advances in next-generation sequencing (NGS), more than 100 genes have been implicated for different types of CMT and other clinically and genetically inherited neuropathies. ${ }^{3,4}$

Widespread clinical and molecular heterogeneity in

*Corresponding Authors: Kimia Kahrizi, MD; Genetics Research Center, University of Social Welfare and Rehabilitation Sciences, Koodakyar Alley, Daneshjoo Blvd., Evin St., Tehran, Iran. Tel: +98-21-22180138; Email: kahrizi@yahoo.com. Hossein Najmabadi, PhD; Genetics Research Center, University of Social Welfare and Rehabilitation Sciences, Koodakyar Alley, Daneshjoo Blvd., Evin St., Tehran, Iran. Tel: +98-21-22180138; Email: hnajm12@yahoo.com \# First authors; contributed equally to this work. 
hereditary neuropathies has created many challenges in the diagnosis of these disorders. Clinical heterogeneity can appear as phenotypic variations (PMP22, GDAP1, SH3TC2, NEFL, IGHMBP2, MME, and DNM2), different modes of inheritance (MFN2, GDAP1, NEFL, $P R X$, and $M P Z)$ or electrophysiological consequences (MPZ, NEFL, GDAP1, and KIF1B). Molecular heterogeneity can be caused by mutations in different genes resulting in phenotypes with similar characteristics (CMT, HMN, and HSAN). ${ }^{5}$

Several mechanisms of molecular and cellular functions are involved in the pathology of CMT and related neuropathies including myelination; membrane and vesicle trafficking; cytoskeletal stability and motor functions; mitochondrial dynamics; RNA processing; protein, lipid and drug metabolism; molecular chaperones; cellular morphology, movement, growth, assembly and maintenance; and cell signaling, death and survival. ${ }^{6}$

To our knowledge, pathogenic alterations in a number of very well-known genes can cause neuropathies mimicking IPN phenotypes, making it difficult to pinpoint a precise diagnosis. ${ }^{1}$

The diagnostic rate for CMT and related IPNs using targeted sequencing including 50 to 80 genes shows a broad spectrum ranging from $19.8 \%$ (Germany) $^{7}$ and $21.6 \%$ (Denmark) $^{2}$ to almost $40 \%$ in studies carried out in France ${ }^{4}$ and $46 \%$ in Norway. ${ }^{8}$

Here, we used whole exome sequencing (WES) to investigate 58 patients with negative results for PMP22 gene dosage alteration to identify the causative variants in known or novel candidate genes.

\section{Material and Methods}

We studied 58 patients with hereditary neuropathy who were referred for molecular diagnosis to the Genetics Research Center (GRC) of University of Social Welfare and Rehabilitation Sciences (USWR) and Kariminejad - Najmabadi Pathology \& Genetics Center (KNPGC) between 2011 and 2018.

Written informed consent, approved by the ethics committees of USWR and KNPGC, was obtained from the patients or their parents. Clinical data with regard to perinatal events, comprehensive family histories, pedigree information and previously performed molecular studies, were collected before WES. Peripheral blood samples from probands, parents and, upon availability, other healthy and/or affected family members were obtained and genomic DNA was extracted using the salting-out method. ${ }^{9}$

In these 58 patients, PMP22 gene deletion/duplication was excluded using the multiplex ligation-dependent probe amplification (MLPA) method (MRC Holland, Netherlands) before WES. Exome enrichment was performed using Agilent Sure Select Human All Exon V5 and V6 enrichment kits (Agilent Technologies Inc, Santa
Clara, CA, USA). The captured libraries were sequenced on Illumina HiSeq 2500 and 4000 platforms (Illumina, San Diego, CA, USA). Following the quality check process using FastQC, the reads were aligned to human genome assembly GRCh37 (hg19) using Burrows-Wheeler Aligner version 0.7.12-r1039. ${ }^{10}$ After trimming the SAM files using Picard tools, the BAM files obtained were subjected to realignment using the GATK pipeline. Subsequently, the haplotype caller module of GATK pipeline was used for variant calling and the variants identified were annotated using the ANNOVAR tool. ${ }^{11}$

The standard analysis pipeline included filtering the frequent variants using public variant frequency databases (filtering out based on MAF>1\%), such as dbSNP151, 1000 Genomes, ${ }^{12}$ ExAC browser, NHLBI GO Exome Sequencing Project and GnomAD browser ${ }^{13}$ as well as the ethnicity-specific database, Iranome (http://www. iranome.ir). ${ }^{14}$ The variants were then prioritized based on gene ontology, clinical significance and bioinformatics prediction scores.

The variants identified were evaluated in terms of compatibility with the patients' clinical phenotype. Sanger sequencing (ABI DNA Sequencer) was applied to validate the candidate variants and investigate their segregation with the disease phenotype in other family members, when practicable. The candidate variants were then classified based on the American College of Medical Genetics and Genomics (ACMG) guidelines. ${ }^{15}$

Following the standard analysis pipeline for 103 genes involved in CMT and related neuropathies (Table S1), analysis of the unresolved cases was continued through investigation of the genes that are either known to contribute in the pathogenesis of neuromuscular disorders ${ }^{16}$ or are highly expressed in peripheral nerves. ${ }^{17}$ Variants within novel genes and other variants of uncertain significance were selected based on the abovementioned criteria, plus the available evidence concerning gene expression and functional studies.

\section{Results}

The investigated patients consisted of 58 unrelated cases with a spectrum of phenotypes and with a preliminary diagnosis of hereditary neuropathies, referred to GRC and KNPGC by physicians and neurologists. The average age for manifestation of the neuropathies was 13 years (range: 4 months to 63 years). Additional phenotypic features were observed in some of the families investigated, as follows: scoliosis (17\%), hearing impairment (8\%), tremor $(5 \%)$ and facial weakness (3\%). All of the cases had been previously ruled out in terms of PMP22 gene dosage alteration using the MLPA method. The clinical electromyography and nerve conduction velocity data were available for 50 out of the 58 cases: $22(37.9 \%)$ patients with demyelinating neuropathy, $21(36.2 \%)$ with axonal neuropathy, $6(10.3 \%)$ with demyelinating sensorimotor 
polyneuropathy with secondary axonal degeneration, and $1(1.7 \%)$ with sensory polyneuropathy and mononeuritis multiplex (Table S2).

In this study, single candidate variants were detected in 52 of the cases, indicating a detection rate of $89.7 \%$. Of the variants identified, 25 were in a homozygous state, 22 in a heterozygous state, and 5 followed an X-linked pattern of inheritance. Using the ACMG guidelines, 27 out of the 52 variants identified were classified as either pathogenic or likely pathogenic, giving a diagnosis rate of $46.6 \%$ (27 out of 58 variants) (Table S3). From the 27 diagnosed cases, 16 had previously known disease-causing variants, and the remaining 11 harbored novel variants in SH3TC2, NEFL, MFN2, MTMR2, ARHGEF10, DNM2, $A T P 1 A 1$ and $H A D H A$ genes.

GJB1 and SH3TC2 were the most frequently mutated genes, each carrying four mutations, and together accounting for $15.4 \%(8 / 52)$ of the 52 cases. Other frequently contributing genes were GDAP1, DNM2, NDRG1, MTMR2, PRX, MME, MFN2, IGHMBP2, NEFL and ARHGEF10. Notably, two identical pathogenic variants, c. $491 \mathrm{G}>\mathrm{A}$ (p.Arg164Gln) in GJB1 and c. $1090 \mathrm{C}>\mathrm{T}$ (p.Arg364*) in PRX genes were observed twice in two unrelated patients.

The 52 single candidate variants, potentially causing the disease phenotypes in the patients, were detected in 35 genes. The great majority of these variants (36/52; 69.2\%) were responsible for CMT and related IPNs. Non-CMT and related IPNs accounted for $21.1 \%(11 / 52)$ of the variants identified, and the remainder $(5 / 52 ; 9.6 \%)$ were linked to the novel candidate genes (Figure 1). Our study revealed phenotypic and genotypic heterogeneity among our investigated patients (Figure 2).

In five of the cases, we identified promising variants within genes (TES, YLPM1, ARHGAPG, SYT8 and $M C A M)$ not previously linked to neuropathies or any

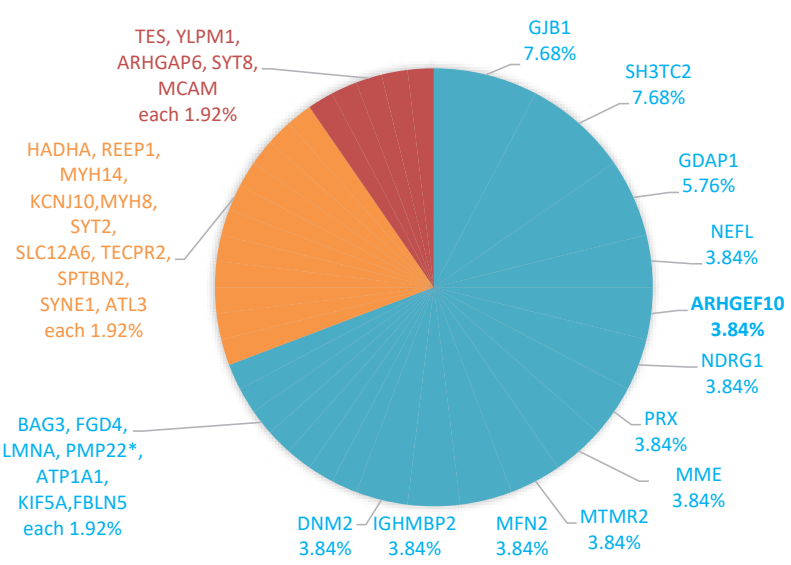

Figure 1. Classification of 52 Single Candidate Variants; blue parts show CMT and related neuropathies, orange parts show non-CMT variants and red parts are related to novel candidate gene variants. PMP22* is a point mutation. established disease phenotypes. Moreover, 10.3\% (6/58) of the cases investigated in this study remained unresolved with either no or two identified candidate variants (Table S3).

\section{Discussion}

IPNs, including CMT as the most common type, represent a large heterogeneous group of hereditary diseases ${ }^{4}$ which perturb peripheral motor and sensory neurons. Considering the high global prevalence of IPN diseases, their genetic diagnosis is of great importance. ${ }^{18}$

In the last few years, the advent of NGS technologies, especially WES, has allowed more comprehensive analysis of heterogeneous single gene diseases including IPNs. ${ }^{19}$ With the help of these new high-throughput technologies, the diagnostic yield for IPNs has improved to over 40\%, ranging from $87 \%$ for CMT1 to $36 \%$ for CMT2. ${ }^{4,8}$

Although our diagnostic rate in this study was $46.6 \%$ $(27 / 58)$, we were able to detect disease-causing variants in 52 out of $58(89.7 \%)$ patients with negative PMP22 gene dosage alteration (Table S3 and S4).

We categorized our detected variants into three gene groups, comprising CMT and related IPN genes, nonCMT and related IPN genes, and novel candidate genes (Table S2).

\section{CMT and Related IPN Genes}

The majority of the disease-causing variants in this study were identified in 19 known CMT and related IPN genes (Figure 1, Table S2A). GJB1 (4/52; 7.7\%) and SH3TC2 $(4 / 52 ; 7.7 \%)$ gene mutations were identified as the genes most frequently causing CMT-related neuropathies in our study.

We observed c.491G>A (p.Arg164Gln) in GJB1, a known pathogenic variant in two out of 58 unrelated patients. The phenotype observed in two patients with

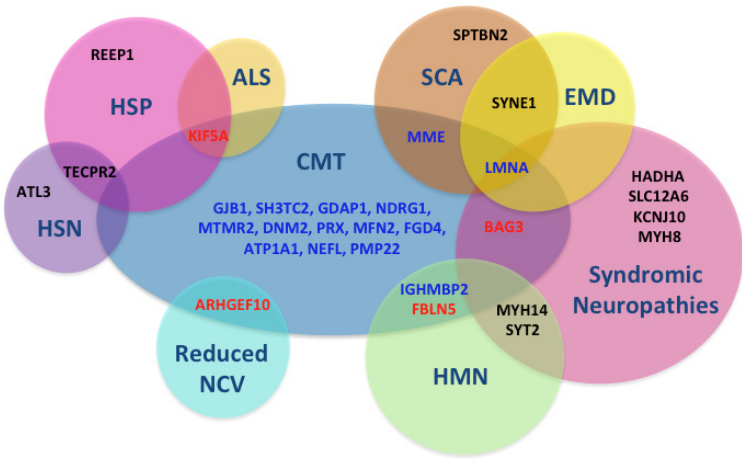

Figure 2. Overlapping Clinical Phenotypes in the Genes Identified Syndromic neuropathies including: Trifunctional protein deficiency, SeSAME syndrome, Agenesis of the corpus callosum with peripheral neuropathy, myofibrillar myopathy, dilated cardiomyopathy, Peripheral neuropathy, myopathy, hoarseness, hearing loss, and Myasthenic syndrome. CMT, Charcot-Marie-Tooth; HMN, hereditary motor neuropathy, HSN, hereditary sensory neuropathy; HSP, hereditary spastic paraplegia; SCA, spinocerebellar ataxia; ALS, amyotrophic lateral sclerosis; EMD, EmeryDreifuss muscular dystrophy. BAG3, FBLN5, ARHGEF10 and KIF5A are genes recently reported in CMT in one or some patients. 
the same mutation showed similar neuromuscular symptoms but hearing impairment was reported in only one patient. Although hearing loss is frequently reported in CMTX patients, two other patients harboring missense mutations in GJB1 also showed no hearing impairment. It is noteworthy that the GJB1 gene has a low rate of benign missense variations, making the missense variants a common mechanism for disease (http://www.hgmd.cf.ac. uk/ac/index.php).

We identified two novel recessive variants, c.233_239delTCTGGGC (p.Leu78Hisfs*49) and c.1366delC (p.Leu456*), in the SH3TC2 gene located at the $\mathrm{N}$-terminus of the protein and between the second $\mathrm{SH} 3$ and first repeat domains, respectively. A significant part of the mutations observed in this gene $(21 \%)$ are related to small deletions (http://www.hgmd.cf.ac.uk/ ac/index.php). In patients with CMT disease, type $4 \mathrm{C}$ (CMT4C) studied by Yger, DiVincenzo and colleagues, the majority of mutations identified showed premature termination codons (PTC) causing specific destruction of the mutated mRNAs by the nonsense-mediated mRNA decay system and loss of function as expected for other PTC mutations in SH3TC2. ${ }^{20,21}$

Senderek et al suggested that all splicing and truncating mutations in the SH3TC2 gene may change the sequence of the longest open reading frame, indicating that a critical amount of the full-length protein is necessary in order to retain a normal phenotype. ${ }^{22}$

Similar to the expected phenotype for CMT4C, caused by mutations in the SH3TC2 gene, the phenotype spectrum in four patients with missense and deletion mutations in this study included muscular weakness and distal muscle atrophy, facial weakness, pes cavus, scoliosis, sensory loss and hearing impairment (Table S4).

We identified two novel dominant variants, c.802_803delTG (p.Trp268Glyfs*22) and c.602A>G (p.Asn201Ser), in the GDAP1 gene located at the GST-C terminus domain. It is very likely that both nonsense and frameshift mutations resulting in truncated proteins lead to GDAP1 loss of function. The GST-C domain is highly conserved and one non-conservative missense mutation will probably result in loss of function by replacing an essential amino acid. ${ }^{23}$ In the case of Asn201Ser alteration, the mutant residue is smaller and more hydrophobic and can result in loss of hydrogen bonds and/or disturbance of correct folding. Moreover, this residue is close to residue 203, which can alter to N6-acetyllysine and interact with G-Cter in ubiquitin. Therefore, the mutation that introduces an amino acid with different properties can disturb the GST C-terminus domain and abolish its function. ${ }^{24}$ Two dominant variations in GDAP1 clinically caused demyelinating neuropathy, lordosis, and areflexia and the recessive variation resulted in axonal CMT (Table S4). From the genotype and phenotype viewpoint, both clinical types comply with the OMIM phenotypes

\section{CMT4A and CMT2K.}

Other frequently mutated genes identified in this study included NEFL, ARHGEF10, NDRG1, PRX, MME, MTMR2, MFN2, IGHMBP2, DNM2, each with a frequency of $3.8 \%(2 / 52)$ (Figure 1, Tables S2A and S4). Disease-causing variations in $B A G 3, F G D 4, L M N A$, PMP22, ATP1A1, KIF5A, and FBLN5 genes were each detected in 1 out of 58 patients investigated in this study (Figure 1).

\section{Non-CMT and Related IPN Genes}

Eleven of the neuropathic variants that we identified causing CMT disease in our patients were genes previously reported in disorders including HSP, spinocerebellar ataxia (SCA), Emery-Dreifuss muscular dystrophy (EMD), and some other neurological inherited syndromes such as congenital myasthenic syndrome, SeSAME (Seizures, Sensorineural deafness, Ataxia, Mental retardation, and Electrolyte imbalance) syndrome, etc. (Figures 1 and 2, Table S2B).

HSP is a heterogeneous group of inherited neurodevelopmental and neurodegenerative conditions resulting from a primary defect in the long descending fibers of the corticospinal tract. ${ }^{25}$ Following the appearance and application of NGS techniques in the identification of genetic causes in this group of diseases, the genetically and clinically overlapping phenotypes between CMT and HSP were emphasized. ${ }^{8,26}$ In this study, we identified variants in HSP-related genes including c.601G $>$ A (p.Ala201Thr) in REEP1 and c.818G $>$ A p.Arg273His in TECPR2 in two individuals referred as neuropathy patients (Table S2B).

The variant in the REEP1 gene was detected in a 55-year-old man, which manifested at the age of 45 and showed muscular weakness and distal muscle atrophy, pes cavus, spastic gait, sensory loss and axonal sensorimotor polyneuropathy (Table S4). The similarity of this patient's symptoms to spastic paraplegia indicates that this person is likely to be affected by spastic paraplegia type 31 (SPG31).

The TECPR2 variant was detected in a 14-yearold female patient with onset at 4 years of age, and manifesting the clinical symptoms of SPG49 including muscular weakness and distal muscle atrophy, spastic gait and chronic demyelinating sensorimotor polyneuropathy (Table S4).

In addition, variants in SCA-related genes were identified in two patients. SCA refers to a group of neurodegenerative disorders (SCA1-40) with progressive cerebellar ataxia and other symptoms resulting from degeneration of the cerebellum and its connections. ${ }^{27}$ This group of diseases can also have phenotypes overlapping with CMT and other neuropathies. ${ }^{28}$ We observed the c.5314G>A (p.Val1772Met) and c.25157C>A (p.Ser8386Tyr) variants in SPTBN2 and SYNE1 genes, respectively, in patients with neuropathic referrals.

In the current study, we found a variant in the SPTBN2 
gene in a 7-year-old female patient with onset at 2 years of age and showing muscular weakness and distal muscle atrophy, ataxic gait, scoliosis, sensory loss and chronic axonal sensorimotor polyneuropathy (Table S4).

A variant in the SYNE1 gene was also identified in a 21-year-old woman with muscular weakness and distal muscle atrophy, pes cavus, sensory loss and cleft palate (Table S4). Mutations in the affected domain (spectrin repeats) have also been reported previously in EMD. ${ }^{29}$

SYT2 is one of the members of the synaptotagmin gene family with different reported mutations in several phenotypes. Presynaptic congenital myasthenic syndrome 7 is the main associated disease and a recent study ${ }^{30}$ has reported a 50-year-old woman with CMT referral and an unusual diagnosis of distal motor neuropathy. We detected a heterozygous variant (c.322A>G; p.Met 108Val) in SYT2 in one of our investigated families manifesting muscular weakness and distal muscle atrophy, pes cavus, hearing loss, sensory loss, and seizure (Table S4).

Mutations in the $H A D H A$ gene have been associated with trifunctional protein deficiency or long-chain 3-hydroxy acyl-coenzyme A dehydrogenase deficiency. In patients affected by distal sensorimotor axonopathy, we detected a novel homozygous, likely pathogenic c.539C > T (p.Pro180Leu) variant. It is noteworthy that $H A D H A$ and $H A D H B$ genes encode the alpha and beta subunits of the mitochondrial trifunctional protein, respectively, and are involved in the fatty acid beta-oxidation pathway which is part of lipid metabolism with some reports of $H A D H B$ mutations in axonal CMT. ${ }^{31}$

Mutations in the SLC12A6 gene cause a spectrum of phenotypic symptoms ranging from agenesis of the corpus callosum to peripheral neuropathy. Our patient with the homozygous c.2075T>G (p.Leu692Arg) variant in this gene had developmental delay, scoliosis, generalized hypotonia, areflexia and demyelinating sensorimotor polyneuropathy (Table S4). Uyanik et al showed that different types of SLC12A6 mutations may cause different clinical symptoms. The classic phenotype of Andermann syndrome is linked to truncated SLC12A6 mutations whereas the phenotype and the course of disease which is associated with missense mutations appeared to be different showing additional features such as diffuse and widespread white matter abnormalities. ${ }^{32}$ Rudnik-Schöneborn et al have also reported a compound heterozygous mutation in the SLC12A6 gene suggesting that mutations of this gene may result in non-syndromic childhood onset HMSN. ${ }^{33}$

SeSAME syndrome arises from KCNJ10 gene mutations. Rarely affected patients show axonal neuropathy and hypomyelination of the sural nerve and one of this study's patients with sensory polyneuropathy and mononeuritis multiplex had a novel homozygous c.967T $>\mathrm{C}$ (p.Tyr323His) variant in the KCNJ10 gene (Table S4).

Mutations in the MYH14 gene cause autosomal dominant hearing impairment and peripheral neuropathy, myopathy, hoarseness and hearing loss. Nerve conduction studies in this type of neuropathy show mildly reduced or normal sensory values. Our patient with the c.3667G>C (p.Gly1223Arg) variant showed muscular weakness and distal muscle atrophy, pes cavus and chronic axonal sensorimotor polyneuropathy with no sign of hearing impairment (Table S4). Choi et al reported a complex phenotype of peripheral neuropathy, myopathy, hoarseness and hearing loss which was linked to an autosomal dominant mutation in MYH14. ${ }^{34}$

The diseases associated with MYH8 include Carney complex variant, arthrogryposis distal type 7 and myopathy distal 1 which is also known as Laing distal myopathy, the afflicted tissues include skeletal muscle and heart, and related phenotypes are high palate and gait disturbance. ${ }^{35}$ We detected a homozygous variant in MYH8 (c.4688G>A, p.Arg1563His) in one of our investigated patients with muscular weakness and distal muscle atrophy, pes cavus, sensory loss and demyelinating sensorimotor polyneuropathy (Table S4).

We detected a heterozygous variant (c.1217G>A, p.Gly406Glu) in the ATL3 gene in one of our investigated patients with muscular weakness and distal muscle atrophy, pes cavus, and lordosis (Table S4). Using WES in a German family with sensory neuropathy and destruction of the pedal skeleton, Kornak et al reported a missense mutation in ATL3, which was a paralog of ATL1, and which was involved in hereditary sensory neuropathy and spastic paraplegia. ${ }^{36}$

\section{Novel Candidate Genes}

In five out of 58 patients, we identified five strong variants in genes not previously linked to any published or identified disease (TES, YLPM1, ARHGAPG, SYT8, and $M C A M)$ (Table S2C).

Synaptotagmin 8 (SYT8) and SYT2, the causative gene for congenital myasthenic syndrome 7 , are a group of membrane-trafficking proteins and can play a role in transmitting neuronal messages, and the subsequent lack of proper function might disrupt synaptic transfers. ${ }^{37}$

SYT8 is expressed in neurons, neuroendocrine and endocrine cells. ${ }^{38}$ We detected a heterozygous variant in SYT8 (c.1099C>T, p. Arg367Trp) (Table S4), which was located at the end of the protein's C-terminus, on the surface of a domain with unknown function after the critical C2 domains; it is thought to be involved in calciumdependent phospholipid binding and in membrane targeting processes such as subcellular localization. ${ }^{38}$

A defect in the SYT1 gene (a homolog of SYT8 human gene) in Drosophila can lead to neuromuscular junction phenotype, ${ }^{39}$ locomotor rhythm defects, neuroanatomical defects and inability to fly. ${ }^{40}$

Testin LIM Domain Protein (TES) is a cytoskeletonassociated focal adhesion scaffold protein that may play a role in cell adhesion, cell spreading, reorganization of the 
actin cytoskeleton and the regulation of cell proliferation. ${ }^{41}$ This protein interacts with a variety of cytoskeletal proteins, such as actin and Actin-like 7A, localizes along actin stress fibers at cell-cell contact areas, and contains three LIM domains. We detected a homozygous variant in TES (c.1161G>C, p. Glu387Asp) (Table S4), in the LIM zinc-binding 3 domain; the double zinc-finger motif functions as adaptors or scaffolds to support the assembly of multimeric protein complexes and mediate proteinprotein interactions between transcription factors, cytoskeletal and signaling proteins. ${ }^{42}$ The small neutral mutant residue in Glu387Asp variant will cause a possible loss of external interactions in this domain. The functional similarity of TES, and proteins interacting with it, to the genes responsible for CMT and other IPNs might justify a role for this gene in causing inherited neuropathies; however, further investigations are required to reveal the precise role of this gene alteration in the manifestation of neuropathy.

YLP Motif Containing 1 (YLPM1) gene is part of cytosol, nucleus and nuclear speck and plays a role in the reduction of telomerase activity during differentiation of embryonic stem cells by binding to the core promoter of telomerase reverse transcriptase and controlling its down-regulation. ${ }^{43}$ We detected a heterozygous variant in YLPM1 (c.5194C>T, p.Arg1732Ter) (Table S4), in one of the patients investigated.

The Zap3 (a homolog of YLPM1 gene) defect has some phenotypes such as developmental dysfunctions in Drosophila melanogaster. ${ }^{44}$

Melanoma Cell Adhesion Molecule's $(M C A M)$ product is involved in anatomical structural morphogenesis, positive regulation of cell migration, angiogenesis, glomerular filtration, cell adhesion and could be an adhesion molecule active in neural crest cells during embryonic development. ${ }^{45}$ We detected a heterozygous splicing variant (loss of the exon 7-end) in the Ig-like C2type 1 domain in MCAM (c.739+2_739+3delTG) (Table S4). Ig-like conserved domains are involved in a variety of functions, including cell-cell recognition, cell-surface receptors, muscle structure and the immune system. ${ }^{46}$

Mutation of this gene's ortholog in mouse leads to an abnormal cytokine level, decreased macrophage cytokine production and decreased angiogenesis, ${ }^{47}$ and in zebrafish, leads to a spectrum of phenotypes such as abnormal somite morphology, epithelial cilium movement involved in the determination of left/right asymmetry phenotype, and axon regeneration phenotype. ${ }^{48}$

Rho GTPase Activating Protein 6 (ARHGAPO) gene's product could regulate the interactions of signaling molecules with the actin cytoskeleton and promotes continuous elongation of cytoplasmic processes during cell motility and simultaneous retraction of the cell body changing the cell morphology. In addition, the ARHGAPG protein is involved in positive regulation of signal transduction, actin filament polymerization and focal adhesion assembly. So, from a functional point of view, this gene is similar to the neuropathy-causing genes. ${ }^{49}$ We detected a homozygous variant ARHGAP6 (c.2236_2237delTC, p. Ser746Glnfs*14) (Table S4) in the main functional Rho-GAP domain. Mutation of this gene's ortholog in mouse leads to abnormal embryonic growth retardation and development. ${ }^{49}$ Sterile females and uncoordinated flies are generated from RhoGAP102A (ortholog of ARHGAPG) gene mutations in Drosophila. ${ }^{50}$

\section{Unresolved Cases}

We failed to detect causative variants in 6 out of 58 $(10.3 \%)$ of our investigated cases (Table S3). In two of the six patients, we identified more than one candidate variant (Table S2D). Homozygous variants c.12329G>A (p.Arg4110His) and c.4732C >T (p.Lue1578Phe) in HSPG 2 and DOCK 6 genes, respectively, were identified in the patient of family ID: 9609011, manifesting muscular weakness and distal muscle atrophy, pes cavus, sensory loss and chronic demyelinating sensorimotor polyneuropathy with secondary axonal degeneration (Table S4).

We identified heterozygous variants c.4066C $>\mathrm{T}$ (p.Arg1356*) and c.1367C>A (p.Ser456*) in MYH15 and NBPF10 genes, respectively, in family ID: 9408104 presenting muscular weakness and distal muscle atrophy, pes cavus and tremor (Table S4).

In conclusion, application of WES in our patients diagnosed with IPN led to the identification of pathogenic and likely pathogenic variants in 27 out of 58 (46.6\%) patients investigated. The reported diagnostic yield of NGS in similar studies with IPN patients ranges from $19.8 \%$ to $46 \% .^{2,4,7,8,51-54}$ The high detection rate of single candidate variants (52 out of 58 patients; $89.7 \%$ ) achieved in this study might reflect the power of WES in genetic diagnostics of CMT-related disorders in consanguineous families harboring no dosage alterations in PMP22 gene.

Our findings expanded the genotype and phenotype spectrum of our patients. Furthermore, novel potential candidate variants identified in this study might increase our understanding of the molecular mechanism underlying IPNs, although further functional studies are still needed to provide insight for the reclassification of variants of uncertain significance detected in this study. Given that most neuropathies are incurable, improving genetic knowledge and diagnostic approaches might help particularly with preventive actions such as carrier detection testing and prenatal diagnosis in families with a positive history of IPN.

\section{Authors' Contribution}

ST, RV, MB, FS, ZF, MM: Technical supports. SA: Families recruitments. $\mathrm{SN}, \mathrm{AK}$ : Clinical supports. HN, KK: Principal investigators.

\section{Conflict of Interest Disclosures}

The authors report no conflict of interest. 
Ethical Statement

Written informed consent, approved by the ethics committees of USWR and KNPGC, was obtained from the patients or their parents in this study.

\section{Acknowledgements}

We would like to express our special thanks to all families for their kind cooperation in this study. This study was supported by the Iran National Science Foundation (INSF) [grant \#: 96011200] to KK and National Institute for Medical Research Development (NIMAD) [grant \#.958744].

\section{Supplementary Materials}

Supplementary file 1 contains Tables S1, S2A, S2B, S2C, S2D and S3. Supplementary file 2 contains Table S4.

\section{References}

1. Mathis S, Goizet C, Tazir M, Magdelaine C, Lia AS, Magy L, et al. Charcot - Marie - Tooth diseases : an update and some new proposals for the classification. J Med Genet. 2015;52(10):68190. doi: 10.1136/jmedgenet-2015-103272.

2. Vaeth $S$, Christensen $R$, Dunø $M$, Lildballe DL, Thorsen $K$, Vissing J, et al. Genetic analysis of Charcot-Marie-Tooth disease in Denmark and the implementation of a next generation sequencing platform. Eur J Med Genet. 2019;62(1):1-8. doi: 10.1016/j.ejmg.2018.04.003.

3. Li LX, Zhao SY, Liu ZJ, Ni W, Li HF, Xiao BG, et al. Improving molecular diagnosis of Chinese patients with Charcot-MarieTooth by targeted next-generation sequencing and functional analysis. Oncotarget. 2016;7(19):27655-64. doi: 10.18632/ oncotarget.8377.

4. Bacquet J, Stojkovic T, Boyer A, Martini N, Audic F, Chabrol B, et al. Molecular diagnosis of inherited peripheral neuropathies by targeted next-generation sequencing: molecular spectrum delineation. BMJ Open. 2018;8(10):e021632. doi: 10.1136/ bmjopen-2018-021632.

5. Drew AP, Zhu D, Kidambi A, Ly C, Tey S, Brewer MH, et al. Improved inherited peripheral neuropathy genetic diagnosis by whole-exome sequencing. Mol Genet Genomic Med. 2015;3(2):143-54. doi: 10.1002/mgg3.126.

6. Timmerman V, Strickland AV, Züchner S. Genetics of CharcotMarie-Tooth (CMT) disease within the frame of the human genome project success. Genes. 2014;5(1):13-32. doi: 10.3390/ genes5010013.

7. Dohrn MF, Glöckle N, Mulahasanovic L, Heller C, Mohr J, Bauer $C$, et al. Frequent genes in rare diseases: panel-based next generation sequencing to disclose causal mutations in hereditary neuropathies. J Neurochem. 2017;143(5):507-22. doi: 10.1111/jnc.14217.

8. Høyer $\mathrm{H}$, Braathen $\mathrm{G}$, Busk $\varnothing \mathrm{L}$, Holla $\varnothing \mathrm{L}$, Svendsen $\mathrm{M}$, Hilmarsen HT, et al. Genetic diagnosis of Charcot-Marie-Tooth disease in a population by next-generation sequencing. Biomed Res Int. 2014;2014:1-13. doi: 10.1155/2014/210401.

9. Miller SA, Dykes DD, Polesky HF. A simple salting out procedure for extracting DNA from human nucleated cells. Nucleic Acids Res. 1988;16(3):1215. doi: 10.1093/nar/16.3.1215.

10. Li H, Durbin R. Fast and accurate long-read alignment with Burrows-Wheeler transform. Bioinformatics. 2010;26(5):58995. doi: 10.1093/bioinformatics/btp698.

11. Wang K, Li M, Hakonarson H. ANNOVAR: Functional annotation of genetic variants from high-throughput sequencing data. Nucleic Acids Res. 2010;38(16):1-7. doi: 10.1093/nar/ gkq603.

12. 1000 Genomes Project Consortium, Auton A, Brooks LD, Durbin RM, Garrison EP, Kang HM, et al. A global reference for human genetic variation. Nature. 2015;526(7571):68-74. doi: 10.1038/nature15393.

13. Karczewski KJ, Francioli LC, Tiao G, Cummings BB, Alföldi J, Wang Q, et al. Variation across 141,456 human exomes and genomes reveals the spectrum of loss-of-function intolerance across human protein-coding genes. bioRxiv. 2019;531210. doi: 10.1101/531210.

14. Fattahi Z, Beheshtian M, Mohseni M, Poustchi H, Sellars E, Nezhadi SH, et al. Iranome: A catalogue of genomic variations in the Iranian population. Hum Mutat. 2019;40(11):1968-84. doi: 10.1002/humu.23880.

15. Richards S, Aziz N, Bale S, Bick D, Das S, Gastier-Foster J, et al. Standards and guidelines for the interpretation of sequence variants: A joint consensus recommendation of the American College of Medical Genetics and Genomics and the Association for Molecular Pathology. Genet Med. 2015;17(5):405-24. doi: 10.1038/gim.2015.30.

16. Bonne G, Rivier F, Hamroun D. The 2019 version of the gene table of neuromuscular disorders (nuclear genome). Neuromuscul Disord. 2018;28(12):1031-63. doi: 10.1016/j. nmd.2018.09.006.

17. VIB Center for Molecular Neurology Faculty. Mutation Database. Inherited Peripheral Neuropathies Mutation Database. Available from http://www.molgen.ua.ac.be/cmtmutations/. Accessed November 2018.

18. Barreto LC, Oliveira FS, Nunes PS, de França Costa IM, Garcez CA, Goes GM, et al. Epidemiologic Study of Charcot-MarieTooth disease: a systematic review. Neuroepidemiology. 2016;46(3):157-65. doi: 10.1159/000443706.

19. Schwarze K, Buchanan J, Taylor JC, Wordsworth S. Are wholeexome and whole-genome sequencing approaches costeffective? A systematic review of the literature. Genet Med. 2018;20(10):1122-30. doi: 10.1038/gim.2017.247.

20. Yger M, Stojkovic T, Tardieu S, Maisonobe T, Brice A, Echaniz-Laguna $A$, et al. Characteristics of clinical and electrophysiological pattern of Charcot-Marie-Tooth 4C. J Peripher Nerv Syst. 2012;17(1):112-22. doi: 10.1111/j.15298027.2012.00382.x.

21. DiVincenzo C, Elzinga CD, Medeiros AC, Karbassi I, Jones JR, Evans MC, et al. The allelic spectrum of Charcot-Marie-Tooth disease in over 17,000 individuals with neuropathy. Mol Genet Genomic Med. 2014;2(6):522-9. doi: 10.1002/mgg3.106.

22. Senderek J, Bergmann C, Stendel C, Kirfel J, Verpoorten N, De Jonghe $\mathrm{P}$, et al. Mutations in a gene encoding a novel $\mathrm{SH} 3$ / TPR domain protein cause autosomal recessive Charcot-MarieTooth type 4C neuropathy. Am J Hum Genet. 2003;73(5):110619. doi: $10.1086 / 379525$.

23. Nelis E, Erdem S, Van Den Bergh PY, Belpaire-Dethiou MC, Ceuterick C, Van Gerwen V, et al. Mutations in GDAP1: autosomal recessive CMT with demyelination and axonopathy. Neurology. 2002;59(12):1865-72. doi: 10.1212/01. WNL.0000036272.36047.54.

24. Venselaar $\mathrm{H}$, te Beek TAH, Kuipers RKP, Hekkelman ML, Vriend G. Protein structure analysis of mutations causing inheritable diseases. An e-Science approach with life scientist friendly interfaces. BMC Bioinformatics. 2010;11(1):548. doi: 10.1186/1471-2105-11-548.

25. Kara E, Tucci A, Manzoni C, Lynch DS, Elpidorou M, Bettencourt C, et al. Genetic and phenotypic characterization of complex hereditary spastic paraplegia. Brain. 2016;139(7):1904-18. doi: 10.1093/brain/aww111.

26. Timmerman V, Clowes VE, Reid E. Overlapping molecular pathological themes link Charcot-Marie-Tooth neuropathies and hereditary spastic paraplegias. Exp Neurol. 2013;246:1425. doi: 10.1016/j.expneurol.2012.01.010.

27. Sun YM, Lu C, Wu ZY. Spinocerebellar ataxia: relationship between phenotype and genotype - a review. Clin Genet. 2016;90(4):305-14. doi: 10.1111/cge.12808.

28. Depondt C, Donatello S, Rai M, Wang FC, Manto M, Simonis $\mathrm{N}$, et al. MME mutation in dominant spinocerebellar ataxia with neuropathy (SCA43). Neurol Genet. 2016;2(5):e94. doi: 10.1212/NXG.0000000000000094.

29. Zhang Q, Bethmann C, Worth NF, Davies JD, Wasner C, Feuer A, et al. Nesprin-1 and -2 are involved in the pathogenesis of Emery - Dreifuss muscular dystrophy and are critical for nuclear envelope integrity. Hum Mol Genet. 2007;16(23):2816-33. doi: 10.1093/hmg/ddm238. 
30. Montes-Chinea NI, Guan Z, Coutts M, Vidal C, Courel S, Rebelo AP, et al. Identification of a new SYT2 variant validates an unusual distal motor neuropathy phenotype. Neurol Genet. 2018;4(6):e282. doi: 10.1212/NXG.0000000000000282.

31. Hong YB, Lee JH, Park JM, Choi YR, Hyun YS, Yoon BR, et al. A compound heterozygous mutation in HADHB gene causes an axonal Charcot-Marie-tooth disease. BMC Med Genet. 2013;14(1):125. doi: 10.1186/1471-2350-14-125.

32. Uyanik G, Elcioglu N, Penzien J, Gross C, Yilmaz Y, Olmez $\mathrm{A}$, et al. Novel truncating and missense mutations of the KCC3 gene associated with Andermann syndrome. Neurology. 2006;66(7):1044-8. doi: 10.1212/01. wnl.0000204181.31175.8b.

33. Rudnik-Schöneborn S, Hehr U, Kalle T Von, Bornemann A, Winkler J, Zerres K. Andermann syndrome can be a phenocopy of hereditary motor and sensory neuropathy report of a discordant sibship with a compound heterozygous mutation of the KCC3 gene. Neuropediatrics. 2009;40(3):129-33. doi: 10.1055/s-0029-1234084.

34. Choi BO, Kang SH, Hyun YS, Kanwal S, Park SW, Koo H, et al. A complex phenotype of peripheral neuropathy, myopathy, hoarseness, and hearing loss is linked to an autosomal dominant mutation in MYH14. Hum Mutat. 2011;32(6):669-77. doi: 10.1002/humu.21488.

35. Oldfors A. Hereditary myosin myopathies. Neuromuscul Disord. 2007;17(5):355-67. doi: 10.1016/j.nmd.2007.02.008.

36. Kornak U, Mademan I, Schinke M, Voigt M, Krawitz P, Hecht J, et al. Sensory neuropathy with bone destruction due to a mutation in the membrane-shaping atlastin GTPase 3. Brain. 2014;137(3):683-92. doi: 10.1093/brain/awt357.

37. Xu Z, Wei G, Chepelev I, Zhao K, Felsenfeld G. Mapping of INS promoter interactions reveals its role in long-range regulation of SYT8 transcription. Nat Struct Mol Biol. 2011;18(3):372-8. doi: 10.1038/nsmb.1993.

38. Monterrat C, Boal F, Grise F, Hémar A, Lang J. Synaptotagmin 8 is expressed both as a calcium-insensitive soluble and membrane protein in neurons, neuroendocrine and endocrine cells. Biochim Biophys Acta. 2006;1763(1):73-81. doi: 10.1016/j. bbamcr.2005.11.008.

39. Herrmann DN, Horvath R, Sowden JE, Gonzalez M, SanchezMejias A, Guan Z, et al. Synaptotagmin 2 mutations cause an autosomal-dominant form of lambert-eaton myasthenic syndrome and nonprogressive motor neuropathy. Am J Hum Genet. 2014;95(3):332-9. doi: 10.1016/j.ajhg.2014.08.007.

40. Loewen CA, Mackler JM, Reist NE. Drosophila synaptotagmin I null mutants survive to early adulthood. Genesis. 2001;31(1):306. doi: 10.1002/gene.10002.

41. Coutts AS, Mackenzie E, Griffith E, Black DM. TES is a novel focal adhesion protein with a role in cell spreading. J Cell Sci. 2003;116(5):897-906. doi: 10.1242/jcs.00278.

42. Dawid IB, Breen JJ, Toyama R. LIM domains: Multiple roles as adapters and functional modifiers in protein interactions.
Trends Genet. 1998;14(4):156-62. doi: 10.1016/S01689525(98)01424-3.

43. Baltz AG, Munschauer $M$, Schwanhäusser B, Vasile $A$, Murakawa Y, Schueler M, et al. The mRNA-bound proteome and its global occupancy profile on protein-coding transcripts. Mol Cell. 2012;46(5):674-90. doi: 10.1016/j.molcel.2012.05.021.

44. Nicholls RE, Gelbart WM. Identification of chromosomal regions involved in decapentaplegic function in Drosophila. Genetics. 1998;149(1):203-215.

45. Kwon O, Miller S, Li N, Khan A, Kadry Z, Uemura T. Bone marrow-derived endothelial progenitor cells and endothelial cells may contribute to endothelial repair in the kidney immediately after ischemia-reperfusion. J Histochem Cytochem. 2010;58(8):687-94. doi: 10.1369/jhc.2010.956011.

46. Teichmann SA, Chothia C. Immunoglobulin superfamily proteins in Caenorhabditis elegans. J Mol Biol. 2000;296(5):1367-83. doi: 10.1006/jmbi.1999.3497.

47. Jiang T, Zhuang J, Duan H, Luo Y, Zeng Q, Fan K, et al. CD146 is a coreceptor for VEGFR-2 in tumor angiogenesis. Blood. 2012;120(11):2330-9. doi: 10.1182/blood-2012-01-406108.

48. Liu CJ, Xie L, Cui C, Chu M, Zhao HD, Yao L, et al. Beneficial roles of melanoma cell adhesion molecule in spinal cord transection recovery in adult zebrafish. J Neurochem. 2016;139(2):187-96. doi:10.1111/jnc.13707.

49. Prakash SK, Paylor R, Jenna S, Lamarche-Vane N, Armstrong DL, $\mathrm{Xu}$ B, et al. Functional analysis of ARHGAP6, a novel GTPaseactivating protein for RhoA. Hum Mol Genet. 2000;9(4):47788. doi: $10.1093 / \mathrm{hmg} / 9.4 .477$.

50. Schinaman JM, Giesey RL, Mizutani CM, Lukacsovich T, SousaNeves R. The KRÜPPEL-like transcription factor DATILÓGRAFO Is required in specific cholinergic neurons for sexual receptivity in drosophila females. PLoS Biol. 2014;12(10):e1001964. doi: 10.1371/journal.pbio.1001964.

51. Laššuthová P, Šafka Brožková D, Krůtová M, Neupauerová J, Haberlová J, Mazanec R, et al. Improving diagnosis of inherited peripheral neuropathies through gene panel analysis. Orphanet J Rare Dis. 2016;11(1):118. doi: 10.1186/s13023-016-0500-5.

52. Nam SH, Hong YB, Hyun YS, Nam da E, Kwak G, Hwang SH, et al. Identification of Genetic Causes of Inherited Peripheral Neuropathies by Targeted Gene Panel Sequencing. Mol Cells. 2016;39(5):382-8. doi: 10.14348/molcells.2016.2288.

53. Yoshimura $A$, Yuan $J H$, Hashiguchi $A$, Ando $M$, Higuchi $Y$, Nakamura T et al. Genetic profile and onset features of 1005 patients with Charcot-Marie-Tooth disease in Japan. J Neurol Neurosurg Psychiatry. 2019;90(2):195-202. doi: 10.1136/jnnp2018-318839.

54. Antoniadi T, Buxton C, Dennis G, Forrester N, Smith D, Lunt $P$, et al. Application of targeted multi-gene panel testing for the diagnosis of inherited peripheral neuropathy provides a high diagnostic yield with unexpected phenotype-genotype variability. BMC Med Genet. 2015;16:84. doi: 10.1186/s12881015-0224-8. 